\title{
Abstammung - oder: Vater, Mutter, Kind gilt nicht mehr!
}

D as reproduktionsmedizinisch Machbare ist in Deutschland eingeschränkt durch das Embryonenschutzgesetz von 1991. Daher lassen viele Paare Behandlungen, die in Deutschland verboten sind (Eizellspende, Leihmutterschaft), im Ausland durchführen. Durch diese Therapien entstehen viele rechtliche Probleme, die nicht ansatzweise gelöst sind.

Sind schon für die Behandlung mit Spendersamen (in Deutschland zulässig und etabliert; zurzeit 1.000 Geburten pro Jahr) erbrechtliche und Unterhaltsprobleme immer noch nicht definitiv geklärt, entstanden neue Probleme durch Entscheidungen höchster deutscher Gerichte. So können die Behandlungen wegen des Grundrechtes auf Kenntnis der genetischen Abstammung nicht mehr anonym durchgeführt werden. Dem kann man ja zustimmen, auch dem Wunsch, dass die so entstandenen Kinder teilweise später einmal Kontakt mit dem genetischen Erzeuger wünschen.

Aber wozu führt die Entscheidung des Bundesgerichtshofes vom 28.1.2015, dass bei berechtigtem Interesse - und das besteht doch wohl immer - die Spenderdaten ohne zeitliche Begrenzung genannt werden müssen? Dieses Urteil führt nach 26 Jahren Untätigkeit des Gesetzgebers zu einer untragbaren rechtlichen Lage und provoziert ein entwicklungspsychologisches Tohuwabohu. Letztendlich wird damit das Modell der Spendersamenbehandlung ad absurdum geführt. Wollte man das? Am besten, das Paar bringt heute seinen eigenen Spender mit und klärt ohne Arzt alle möglichen rechtlichen Implikationen.

Eizellspenden sind in Deutschland verboten, aber geschätzt 1.000 Paare lassen eine entsprechende Behandlung erfolgreich im Ausland durchführen.
„Mutter ist, wer das Kind zur Welt bringt!“ Abgesehen von ethisch-moralischen Problemen (s. aktueller Fall einer 65-jährigen, ledigen Frau mit Vierlingen nach Behandlung in der Ukraine unter Missachtung aller deutschen Regeln für die Fortpflanzungsmedizin) besteht für das Ergebnis dieser Behandlung keine Gleichbehandlung in Bezug auf die Samenspende. Die nach Eizellspende geborenen Kinder haben bisher keine Möglichkeit, ihre genetische Abstammung in Erfahrung bringen zu können. Und noch abwegiger wird es, wenn ein männliches homosexuelles Paar ein Kind durch eine Leihmutter zum Beispiel in Indien oder Kalifornien austragen lässt und gesetzwidrig in Deutschland die Vaterschaft zweier Väter eingetragen wird, obwohl Leihmutterschaft verboten ist.

Vater, Mutter, Kind gilt nicht mehr: Kinder können eine Vielzahl von Eltern haben, genetische Eltern, Eizellspenderinnen, Samenspender, Leimutter oder Eltern, die es aufziehen. Wem gebührt das Sorgerecht? Wer ist unterhaltspflichtig? Für wen wird das Kind Sorge tragen müssen? Von wem kann es erben? Das deutsche Recht kennt auf viele dieser Fragen keine Antworten. Die Rechtslage in Deutschland beim Abstammungsrecht und in der Reproduktionsmedizin läuft der internationalen Entwicklung hinterher. Die im Koalitionsvertrag vereinbarte Arbeitsgruppe „Modernisierung des Abstammungsrechts" beim Justizministerium hat noch keine brauchbaren Vorschläge am Ende der Legislaturperiode geliefert.

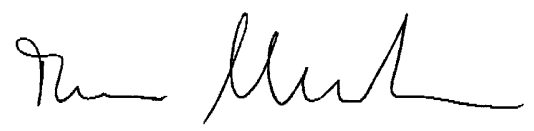

